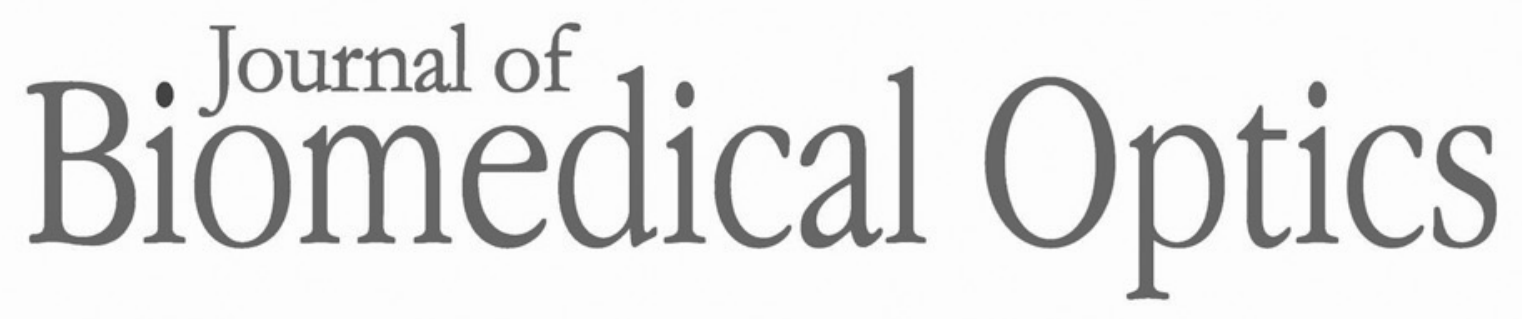

BiomedicalOptics.SPIEDigitalLibrary.org

\title{
2018 List of Reviewers
}


The Journal of Biomedical Optics would like to sincerely thank the following individuals who served as reviewers in 2018. The success of our publication hinges on the voluntary contributions of time and energy put forth by these professionals.

\begin{tabular}{|c|c|c|}
\hline Ousama A'Amar & Emmanuel Bossy & Anabela Da Silva \\
\hline Latifa Abdelgawad & Richard Bouchard & Chris Dainty \\
\hline Ibrahim Abdulhalim & Nada Boustany & Maxim Darvin \\
\hline Nicole Ackerman & Audrey Bowden & Hadis Dashtestani \\
\hline Robert Adamson & Jonathan Brand & Rupsa Datta \\
\hline Steven Adie & Nikolay Brandt & Digant Dave \\
\hline Yoshihisa Aizu & Jaime Bravo & Richard Day \\
\hline Visar Ajeti & Matthew Brenner & Helga De Oliveira Miguel \\
\hline Dennis Alexander & Edward Brown & Frederico de Sousa \\
\hline Robert Alfano & J. Quincy Brown & Xose Luis Dean Ben \\
\hline Alba Alfonso-Garcia & Joanna Brunker & Anthony Deegan \\
\hline Marc Antoine Allard & Erin Buckley & Hamid Dehghani \\
\hline David Alonso-Caneiro & Anne-Laure Bulin & James Delikatny \\
\hline Robert Amelard & Takashi Buma & Nikolaos Deliolanis \\
\hline Zahra Amini & Peter Burgholzer & Valentin Demidov \\
\hline Marine Amouroux & Andrew Burnett & Bin Deng \\
\hline Franck Amyot & David Busch & Kurt Denninghoff \\
\hline Suresh Anand & Sandra Bussadori & Michele Diana \\
\hline Afrouz Anderson & Fuhong Cai & Alberto Diaspro \\
\hline Stefan Andersson-Engels & Acner Camino Benech & Jouke Dijkstra \\
\hline Joseph Angelo, Jr. & Andrew Camp & Galina Dimitrova \\
\hline Oleg Angelsky & Kirby Campbell & Yichen Ding \\
\hline Bhavna Antony & Murat Canpolat & Zhihua Ding \\
\hline Matthew Applegate & Rui Cao & U.S. Dinish \\
\hline Kavita Arora & Stefan Carp & Mamadou Diop \\
\hline Hossein Asghari & Oscar Carrasco-Zevallos & Ana Doblas \\
\hline Marc Aubreville & Jefferson Chan & Zafer Dogan \\
\hline Marco Augustin & Chien-Ming Chen & Chen-Yuan Dong \\
\hline Yasuhiro Awatsuji & Jianxin Chen & Alexander Doronin \\
\hline Mehdi Azimipour & Nanguang Chen & Tatjana Dostalova \\
\hline Justin S. Baba & Shih-Chi Chen & Alexandre Douplik \\
\hline Saulius Bagdonas & Sung-Liang Chen & Tanja Dragojevic \\
\hline Yuquiang Bai & Wanyi Chen & Patrick Drew \\
\hline Wesley Baker & Wei Chen & Bruce Drum \\
\hline Gemma Bale & Wei-Liang Chen & Dane Drutis \\
\hline Lucia Ballerini & Wenlung Chen & Can Duan \\
\hline Mihaela Balu & Yu Chen & Suzie Dufour \\
\hline Timothy Baran & Zhongping Chen & Nicholas Durr \\
\hline Chris Barker & Parag Chitnis & Pranab Dutta \\
\hline Margarida Barroso & Hak Soo Choi & Reza Ebrahimpour \\
\hline Jennifer Barton & Woo Choi & Janis Eells \\
\hline Alexey Bashkatov & Ken Chu & Adam Eggebrecht \\
\hline Aakash Basu & Joseph Chue-Sang & Jonathan Elliott \\
\hline Adam Bauer & Esther Ciarrocchi & Daniel Elson \\
\hline Paul Beard & Riccardo Cicchi & Mohsen Erfanzadeh \\
\hline Kathy Beaudette & Michal Cifra & Marica Ericson \\
\hline Kate Bechtel & Jeffrey Cochran & Sergey Ermilov \\
\hline Andrey Belikov & Danut Adrian Cojoc & Rafael Espinosa-Luna \\
\hline Muyinatu Bell & Jean-Luc Coll & Conor Evans \\
\hline Mikhail Berezin & Olga Conde & Dirk Faber \\
\hline Karla Bergonzi & Emma Condy & David Fahd \\
\hline Tiziano Binzoni & Daniel Cote & Andrew Fales \\
\hline Judith Birkenfeld & Brian Crouch & Xudong Fan \\
\hline Renata Bitar & John H. Crowe & Zhichao Fan \\
\hline Kostadinka Bizheva & Brian Cullum & Qianqian Fang \\
\hline Richard Blackmon & Brian Culshaw & Felix Fanjul-Velez \\
\hline Walter Blondel & Andrea Curatolo & Sergio Fantini \\
\hline Nienke Bosschaart & Cosimo D'Andrea & Parisa Farzam \\
\hline
\end{tabular}


Baowei Fei

Reza Fekrazad

Jinchao Feng

Shangyuan Feng

Xu Feng

Farzad Fereidouni

Jonathan Fisher

Maryann Fitzmaurice

James Fitzpatrick

Kayvan Forouhesh Tehrani

Walfre Franco

Ingemar Fredriksson

Daniel Fried

Nathaniel Fried

Ling Fu

Thilo Gambichler

Yu Gan

Israel Gannot

Alex Gao

Feng Gao

Hao Gao

Peter Gardner

Jiajia $\mathrm{Ge}$

Valentin M. Gelikonov

Irene Georgakoudi

Pejman Ghassemi

Adrian Ghita

Nirmalya Ghosh

Elisabetta Giorgini

Martina Giovannella

Randolph Glickman

Arthur Gmitro

Keisuke Goda

Dimitris Gorpas

Will Goth

Clara Gregori-Pla

Kate Grieve

Dirk Grosenick

Viktor Gruev

Ireneusz Grulkowski

Maxime Guinin

Murat Gulsoy

Jacqueline Gunther

Kaikai Guo

Steffen Hackbarth

Pengfei Hai

Bertan Hallacoglu

Kimberly Hamad-Schifferli

Michael Hamblin

Martin Hammer

Songfeng Han

Anderson Hara

Danielle Harper

Andrew R. Harvey

Andreas Hauptmann

Joseph Hayward

Michal Heger

Herbert Heise

Morgan Heisler

Marjaneh Hejazi
Maged Henary

Christine Hendon

Sophie Hernot

Alison Hobro

Jon Holmes

Roarke Horstmeyer

Vladimir Hovhannisyan

Hsun-Chia Hsu

Song $\mathrm{Hu}$

Jiwei Huang

Zheng Huang

Gereon Huettmann

Jie Hui

Sam Hutchings

Mikko Huttunen

Masatoshi Ichikawa

Meltem Izzetoglu

Steven Jacques

Mooseok Jang

Gregor Jemec

Minbiao Ji

Mengyu Jia

Shudong Jiang

Shuliang Jiao

Joseph Jing

Jami Johnson

Jesse V. Jokerst

James Joseph

Vinayak Joshi

Jana Kainerstorfer

Vyacheslav Kalchenko

Jeroen Kalkman

Sadao Kaneko

Hyun Wook Kang

Jeon Woong Kang

Jin Kang

Rajan Kanhirodan

Dimitris Kapsokalyvas

Rahul Kavi

Adib Keikhosravi

Matthew Keller

Gordon Kennedy

Kelsey Kennedy

David Kessel

Morteza Khaleghi

Siddharth Khare

Alwin Kienle

Arnold Kim

Chulhong Kim

Jeehyun Kim

Ki Hean Kim

Sean J. Kirkpatrick

Justin Klein

Matthias Klemm

Alex Ko

Naoki Kobayashi

Irene Kochevar

Radim Kolar

Kivanc Kose

Sri-Rajasekhar Kothapalli
Christoph Krafft

Joerg Krueger

Anthony Kuo

Wen-Chuan Kuo

Roman Kuranov

Salim Lahmiri

Joseph LaManna

Pierre Lane

Kirill Larin

Irina Larina

Ethan LaRochelle

Jan Laufer

Marcel Lauterbach

Antonio Lauto

Frederic Leblond

Igor Lednev

Byeong Ha Lee

Chang Lee

Hsiang-Chieh Lee

Robert Lee

Woei Ming Lee

Anais Leproux

Aymeric Leray

Richard Levenson

Biyuan Li

Changhui Li

Changqing $\mathrm{Li}$

Chunqiang $\mathrm{Li}$

Hai Li

Jiao Li

Jiasong $\mathrm{Li}$

Jiawen $\mathrm{Li}$

Lei Li

$\mathrm{Li} \mathrm{Li}$

Peng Li

Qingli Li

Weibo Li

Xinge $\mathrm{Li}$

Zhifang $\mathrm{Li}$

Zongxi Li

Rongguang Liang

Wenxuan Liang

Lothar Lilge

Hee Lim

Wei-Chiang Lin

Tom Lister

Fei Liu

Gangjun Liu

Hanli Liu

Linbo Liu

Peng Liu

Quan Liu

Shengnan Liu

Tzu-Ming Liu

Xuan Liu

Giuseppe Lo Presti

Fake Lu

Guolan Lu

Rongwen Lu

Holger Lubatschowski 
Felix Lucka

Geoffrey Luke

Sarah Lukes

Jianwen Luo

Cheng $\mathrm{Ma}$

Hui Ma

Samuel Mabbott

Lewis MacKenzie

Anita Mahadevan-Jansen

Boris Majaron

Shuichi Makita

Benjamin Maloney

Zbignevs Marcinkevics

Haley Marks

Fabrizio Martelli

Vadivel Masilamani

Vasily Matkivsky

Lev Matveev

David Mayerich

Avraham Mayevsky

Amaan Mazhar

David McClatchy

David McGloin

Robert McLaughlin

Lacey McNally

Andrei Medvedev

Igor Meglinski

Martina Meinke

Joerg Meister

Zhaokai Meng

Sabina Merlo

Andre Mermoud

Jerome Mertz

Diana Messadi

Kirk Michaelian

J. Mieog

Daniel Milej

Takeo Minamikawa

Leonidas Mindrinos

Ashish Kumar Mishra

Kenji Mitsuhashi

Andreia Moco

Suman Mondal

Inkyu Moon

Jason Moore

Joshua Morgan

Alexander Moskalensky

Judith Mourant

Meghdoot Mozumder

Peter Munro

Moussa N'gom

Annemarie Nadort

Oleg Nadyarnykh

M. Nasiriavanaki

Shubhankar Nath

Rodrigo Nava

Daniel Neal

Meda Negrutiu

John Quan Nguyen

Phuc Nguyen
Thanh Nguyen

M. Khalid Khan Niazi

Brandon Nichols

Mark Niedre

Anna-Liisa Nieminen

Bo Ning

Shoko Nioka

Nozomi Nishimura

Meir Nitzan

Haijing Niu

Teruyoshi Nobukawa

Jiri Novak

Sergio Novi

Tatiana Novikova

Robert Nuster

Matthew O'Donnell

Dennis O'Neal

Thomas O'Sullivan

Yusuke Ogura

Jun Ohta

Eiji Okada

Luis Oliveira

Malini Olivo

Nobuhiro Ooba

Cynthia Pagba

Hamid Pahlevaninezhad

Santosh Paidi

Sven Painer

Marco Palanca

Nikhil Paliwal

Guenther Paltauf

Rishikesh Pandey

Sorin Viorel Parasca

Hyeon-Cheol Park

Je-Kyun Park

YongKeun Park

Nimit Patel

Ronak Patel

Lynn Paterson

Ajeetkumar Patil

Rusha Patra

Yannis Paulus

Francesco Pavone

Ivan Pelivanov

Giovanni Pellacani

Kara Peters

Elena Petrova

Giuseppe Pezzotti

T. Pfefer

Jennifer Phipps

Daqing Piao

Emma Pickwell-MacPherson

Angelo Pierangelo

Mark Pierce

Roberto Pini

Nancy Pleshko

Andrew Plumb

Brian Pogue

Daria Pominova

Chien Sing Poon
Alexey Popov

Juergen Popp

Eric Potma

Prabhakar Pradhan

Scott Prahl

Jaya Prakash

Manojit Pramanik

Stefan Preisser

Alexander Priezzhev

Ji Qi

Xiaoli Qi

Haohua Qian

Junle Qu

Xiangyu Quan

Raquel Queiros

Kyle Quinn

Narasimhan Rajaram

Jessica Ramella-Roman

Evelio E. Ramirez-Miquet

Julio Ramirez-San Juan

Chongzhao Ran

Lise Randeberg

Mahsa Ranji

Christopher Raub

Aniruddha Ray

Daniel Razansky

Rebecca Re

Nicholas Reder

Karen Reiser

Andrew Resnick

Jungtae Rha

Rebecca Richards-Kortum

Charles Riva

William Roach

K. Thomas Robbins

David Roberts

Darren Roblyer

Peter Rolfe

Jannick Rolland

Emilie Roncali

E. Victor Ross

Vincent Rossi

Ricardas Rotomskis

Sidheswar Routray

Steven Rowe

Florian Rudnitzki

Eduardo Ruvolo

Ramkumar Sabesan

Paola Saccomandi

Alireza Sadr

Peeyush Sahay

Amit Sahu

Partha Sahu

Ranjit Sahu

Sava Sakadzic

Ahmad Salman

Ota Samek

Masood Samim

Danuta Sampson

Maria Sanchez-Lopez 
Camila Santos

Marinko Sarunic

Angelo Sassaroli

Shunichi Sato

Firas Sawaf

Ilyas Saytashev

Giuliano Scarcelli

Ronald Schachar

Tilman Schmoll

Herbert Schneckenburger

Felix Scholkmann

Richard Schwarz

Dietrich Schweitzer

Eric Seibel

Oxana S.-Glushkovskaya

Oleg Semyonov

Inseok Seo

Yu Shang

Peng Shao

Calvin Shaw

Vladislav Shcheslavskiy

Benjamin Sherlock

Fei Shi

Lingyan Shi

Wei-Chuan Shih

Dustin Shipp

Marina Shirmanova

Xiao Shu

Mira Sibai

Adam Sibley

Ludovico Silvestri

Surya Singh

Abhijit Sinha Roy

Kenneth Sloan

Jason Smith

Venugopal Rao Soma

Liang Song

Shaozhen Song

Foo Chong Soon

Jarich Spliethoff

Vivek Srinivasan

Ronald Sroka

Georgios Stamatas

Idan Steinberg

Herbert Stepp

Henricus Sterenborg

Philipp Steven

Chiara Stringari

Tomas Stromberg

Rodrigo Suarez

Pavel Subochev

Klaus Suhling

Aaron Suminski

Xin Sun

Ulas Sunar

Kung-Bin Sung

Nima Tabatabaei

Johnny Tam

Bingyao Tan

Xin Tan
Jianbo Tang

Qinggong Tang

Yubo Tang

Patrice Tankam

Irwin Tendler

Suresh Thennadil

Simon Thibault

Chao Tian

Jie Tian

Limei Tian

Kenneth Tichauer

Karissa Tilbury

Philip Tinnefeld

Yunjie Tong

Mohammad Torabzadeh

Vladislav Y. Toronov

Kimani Toussaint

Markandey Tripathi

Chia-Ling Tsai

Vassiliy Tsytsarev

Esra Tunc Gormus

Giovanni Ughi

Hafeez Ullah

Sergey Ulyanov

Paul Urayama

Urs Utzinger

Ram Vaderhobli

Pablo Valdes

Niksa Valim

Petri Valisuo

Alexey Valkov

Fons van der Sommen

Martin van Gemert

Ton van Leeuwen

Martin van de Ven

Jenu Varghese Chacko

Fartash Vasefi

Fabrice Vaudelle

Hamed Vavadi

Pedro Vaz

Rudolf Verdaasdonk

Martin Villiger

Phuong Vincent

Karthik Vishwanath

I. Alex Vitkin

Steven Vogel

Valentine Vullev

Sunil Vyas

Nilesh Wagh Wagh

Georges Wagnieres

Horst Wallrabe

Alexandra Walsh

Hao Wang

Hui Wang

Jincheng Wang

Lidai Wang

Lin Wang

Quan Wang

Quanzeng Wang

Shang Wang
Thomas Wang

Tianyi Wang

Xueding Wang

Yu Wang

Yuguang Wang

Zhao Wang

Jason Warram

Andreas Wartak

Wataru Watanabe

Jessie Weber

Robert Weersink

Xunbin Wei

Wendy Wells

Julia Welzel

Paul S. Westbrook

Ryan White

Petra Wilder-Smith

Brian Wilson

Jesse Wilson

Dennis Wirth

Stanislaw Wojtkiewicz

Kenneth Kin-Yip Wong

Terence Wong

Binlin Wu

Jia Wu

Jianglai Wu

Yicong Wu

Lara Wurster

Lei Xi

Jun Xia

Wenfeng Xia

Liangzhong Xiang

Huikai Xie

Tuqiang Xie

Chen Xu

Guan Xu

$\mathrm{He} X u$

Min Xu

Yi Xue

Amir Yadegari

Tatsuyuki Yamamoto

Bin Yang

Muhan Yang

Xinmai Yang

Gang Yao

Junjie Yao

Zahid Yaqoob

Mohammad Yaseen

Yoshiaki Yasuno

Alvin Yeh

Ji Yi

Biwei Yin

Xin Yin

Hongki Yoo

Tadayuki Yoshitake

Lingfeng $\mathrm{Yu}$

Ping $Y u$

Jie Yuan

Shuhua Yue

Vladimir Zaitsev 
Valeriy Zakharov Elisabetta Zanetti Jason Zara

Mark Zarella

Cristina Zavaleta

Randa Zein

Haishan Zeng

Shaoqun Zeng

Guanglei Zhang

Hao Zhang

Qinqin Zhang

Quan Zhang

Yanbo Zhang
Hubin Zhao

Jianhua Zhao

Shuang Zhao

Yanyu Zhao

Guoan Zheng

Wei Zheng

Zhenrong Zheng

Chao Zhou

Liangcheng Zhou

Qifa Zhou

Renjie Zhou

Banghe Zhu

Caigang Zhu
Dan Zhu

Jiang Zhu

Liren Zhu

Quing Zhu

Yizheng Zhu

Peyman Zirak

Leonid Zubkov

Andres F. Zuluaga

Fernando Zvietcovich

Andrei Zvyagin

Andrey Zyubin 